\title{
Téoros
}

Revue de recherche en tourisme

\section{Entre mer et climat}

\section{Le cas de la Colombie-Britannique}

\section{Jean Stafford et Bruno Sarrasin}

Volume 20, numéro 1, printemps 2001

Mers et littoraux

URI : https://id.erudit.org/iderudit/1071906ar

DOI : https://doi.org/10.7202/1071906ar

Aller au sommaire du numéro

Éditeur(s)

Université du Québec à Montréal

ISSN

0712-8657 (imprimé)

1923-2705 (numérique)

Découvrir la revue

\section{Citer cet article}

Stafford, J. \& Sarrasin, B. (2001). Entre mer et climat : le cas de la

Colombie-Britannique. Téoros, 20(1), 17-21. https://doi.org/10.7202/1071906ar

Ce document est protégé par la loi sur le droit d'auteur. L'utilisation des services d'Érudit (y compris la reproduction) est assujettie à sa politique d'utilisation que vous pouvez consulter en ligne.

https://apropos.erudit.org/fr/usagers/politique-dutilisation/
Cet article est diffusé et préservé par Érudit.

Érudit est un consortium interuniversitaire sans but lucratif composé de l'Université de Montréal, l'Université Laval et l'Université du Québec à Montréal. Il a pour mission la promotion et la valorisation de la recherche. https://www.erudit.org/fr/ 


\section{Entre mer et climat Le cas de la Colombie-Britannique}

Jean Stafford et Bruno Sarrasin

\author{
La Colombie-Britannique offre plus \\ de 700 kilomètres de côte escarpée \\ qui longent le Pacifique. Son long \\ littoral est parsemé d'îles et de \\ frords très accidentés. Son climat est \\ tempéré ide $18^{\circ} \mathrm{C}$ à $20^{\circ} \mathrm{C}$ l'été et \\ de $0^{\circ} \mathrm{C}$ à $2{ }^{\circ} \mathrm{C}$ l'hiver) et toute la \\ zone côtière est réchauffée par les \\ courants de l'océan Pacifique. Ses \\ deux principales villes, Vancouver \\ et Victoria, sont situées dans cette \\ zone côtière à tout juste 100 kilo- \\ mètres l'une de l'autre.
}

8 ur le plan économique, $68 \%$ des ex portations de la Colombie-Britannique vont vers les Etats-Unis, $15 \%$ vers le Japon, $7 \%$ vers d'autres pays d'Asie (Corée, Taïwan, Hong Kong, Chine), $6 \%$ vers l'Europe et $4 \%$ vers le reste du monde $\mathrm{w}^{\sharp}$. L'économie de cette province, comme l'économie de l'ensemble des provinces canadiennes, est fortement intégrée à l'économie américaine ; l'influence du Japon et de certains pays d'Asie se fait aussi sentir:

L'industrie touristique représentait, en $1998,4,8 \%$ du PIB, soit 4292 milliards de dollars (en dollars constants de 1992). En 1999, 22,3 millions de touristes ont visité la Colombie-Britannique dont les. principales activités touristiques sont axées sur le plein air et les visites de sites naturels. Le littoral est au centre de l'offre touristique de la province. Il suffit de penser aux îles de Victoria et de Vancouver ainsi qu’aux activités de villégiature et aux croisières qui y sont proposées pour saisir l'ampleur de l'attrait qu'exerce la mer. Au-delà des plages familiales ou isolées. la Colombie-Britannique propose un nombre important de sentiers pédestres en bord de mer, comme celui de la West Coast Trail situé sur l'île de Victoria. Aménagé au tournant du siècle dernier pour permettre aux naufragés d'atteindre la civilisation, ce sentier de 77 kilomètres est le parfait exemple du positionnement de la Colombie-Britannique : entre mer, nature et montagne.

\section{L'évolution du tourisme depuis 1972}

Le tourisme en Colombie-Britannique a connu, de 1972 à 1999 , une croissance très forte, comme le dérmontre le graphique 1. De 1972 à 1982, on note une certaine stagnation des arrivees de touristes. C'est l'Exposition universelle de 1986 qui a été le détonateur de la croissance. On remarque aussi que, de 1983 à 1999 , la croissance des arrivées de touristes est quasi exponentielle.

Le tableau 1 montre le taux d'accroissement annuel moyen (moyenne géométrique) des arrivées de touristes, par blocs de cinq années, pour les touristes en provenance des États-Unis et des autres pays. de 1980 à 1999.

Le taux d'aceroissement des touristes américains progresse graduellement pour les trois périodes étudiées. Les arrivées des visiteurs des autres pays (à l'exception des États-Unis), à l'inverse, diminuent graduellement (après une très forte expansion en 1985-1989) pendant les mêmes périodes.

\section{L'éfude des clientèles - les parts de marché}

En 1972, les parts de marché des touristes en provenance des Etats-Unis étaient de $97 \%$; en 1999 , le pourcentage était de $83 \%$ pour les Etats-Unis et de $17 \%$ pour les autres pays. Cette évolution du secteur touristique correspond à celle des autres secteurs économiques durant cette même période. En 1972, l'Europe détenait $49 \%$ des parts de marché, l'Asie $34 \%$, l'Océanie $10 \%$ et les autres continents $7 \%$ de l'ensemble. En l'an 2000, les proportions passaient à $35 \%$ pour l'Europe, $50 \%$ pour I'Asie, $9 \%$ pour l'Occanie et $6 \%$ pour les autres continents. En Europe, pres de $70 \%$ des clientèles proviennent du RoyaumeUni et de l'Allemagne; en Asie, $7 \%$ des touristes viennent de quatre principaux 


\section{TABLEAU}

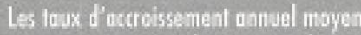
(rivyean: geonatriquel des criveses de touistes an Co or hie-Bribentiqse, de 1980 a 1999 lea $\mathrm{X}$ )

\begin{tabular}{|c|c|c|c|c|c|c|}
\hline ANNÉES & \multicolumn{2}{|c|}{ ËTATSUUIS } & \multicolumn{2}{|c|}{ AUTRES PASS } & \multicolumn{2}{|c|}{ TOTAL } \\
\hline 1980-1984 & $-0,7$ & \multirow[t]{2}{*}{1,2} & 1,9 & \multirow[t]{2}{*}{7,4} & $-0,4$ & \multirow[t]{2}{*}{24} \\
\hline $1985-1989$ & 2,7 & & 16,9 & & 4,2 & \\
\hline $1990-1994$ & 3,4 & \multirow[t]{2}{*}{5,0} & 7,5 & \multirow[t]{2}{*}{7,7} & 3,9 & \multirow[t]{2}{*}{5,4} \\
\hline $1995-1999$ & 6,3 & & 3,9 & & 5,9 & \\
\hline $1980-1999$ & \multicolumn{2}{|c|}{34} & \multicolumn{2}{|c|}{7,5} & \multicolumn{2}{|c|}{3,9} \\
\hline
\end{tabular}

Source: Gouvernemenil de lo Colombie-Beitanique.

pays : Japon, Taiwan, Hong Kong et Coree du Sud. Il s'agit donc de clientèles très concentrées dans certains pays de ces deux continents.

Les parts de marché de l'Europe et de l'Asie fluctuent considerablement de 1972 à 2000 et suivent gếnéralement l'évolution des conjonctures Économiques durant cette période. La Colombie-Britannique est une des rares provinces canadiennes dont l' économie et le tourisme sont aussi diversifiés.

De 1972 à 1984 , le continent européen détenait près de $50 \%$ des parts de marché : après 1988 , on note un renversement de cette tendance au profit du continent asiatique. En 1997, les visiteurs en provenance d'Asie représentaient $56 \%$ des clientèles Etrangères (a l'exception des Etats-Unis). En l'an 2000, la situation qui prévalait en 1972 est complètement inversée : le continent europeen regroupe $35 \%$ des parts de marché et le continent asiátique $50 \%$. Les autres continents restent tous sous la barre des $10 \%$ des parts de marché du tourisme en Colombie-Britannique.

\section{L'évolution à long terme des principales clientèles}

Nous avons vu que la clientèle touristique en provenance des Etats-Unis est de loin la plus importante. Depuis 1995 , 1a progression a ếté assez forte. Le modèle prévisionnel (une tendance quadratique) suppose un taux d'accroissement annuel moyen de 4,1 \% de 2000 à 2005 (voir le graphique 2).

Les arrives de touristes du continent européen devraient connaître une croissance

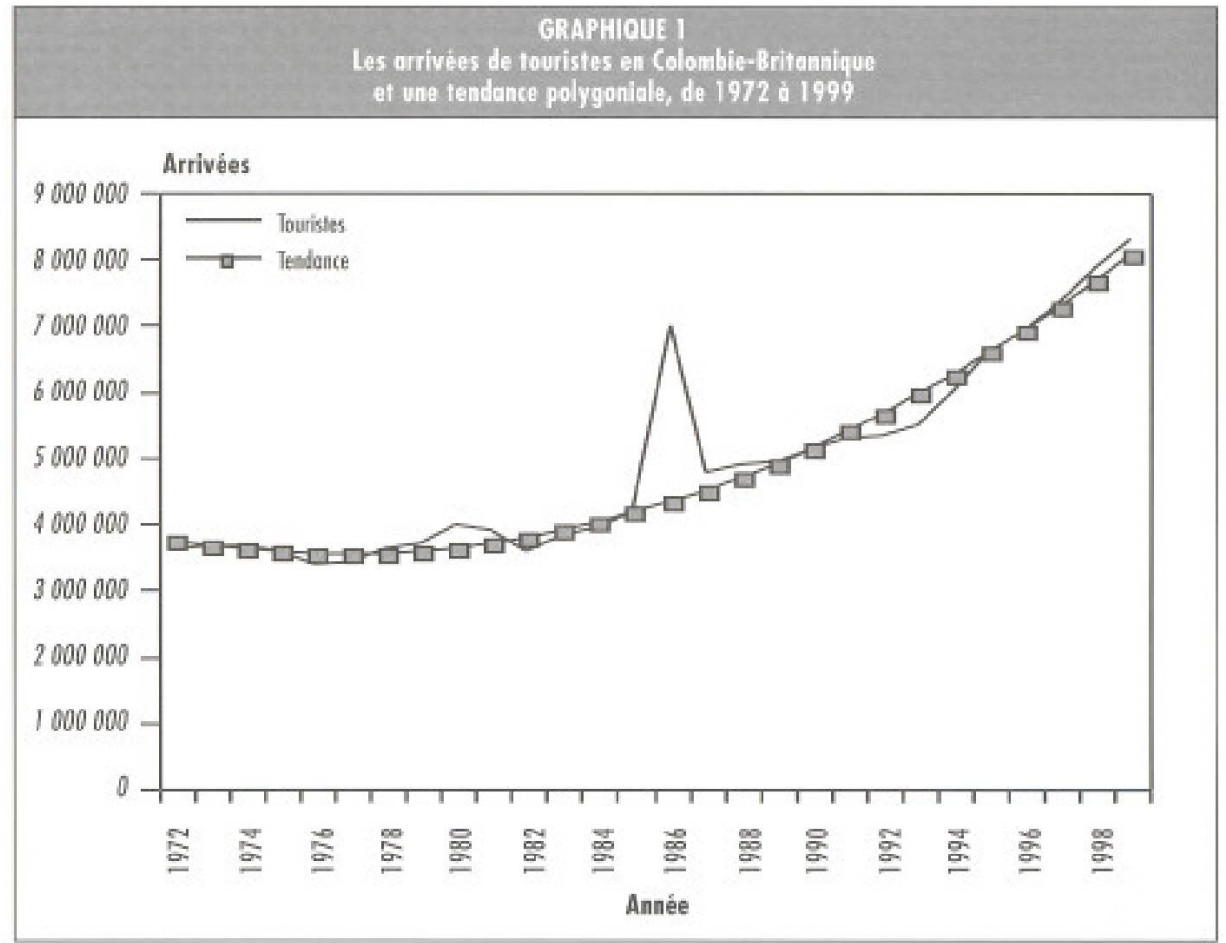

Source : Gomamement de lo Colombie-Brikanique

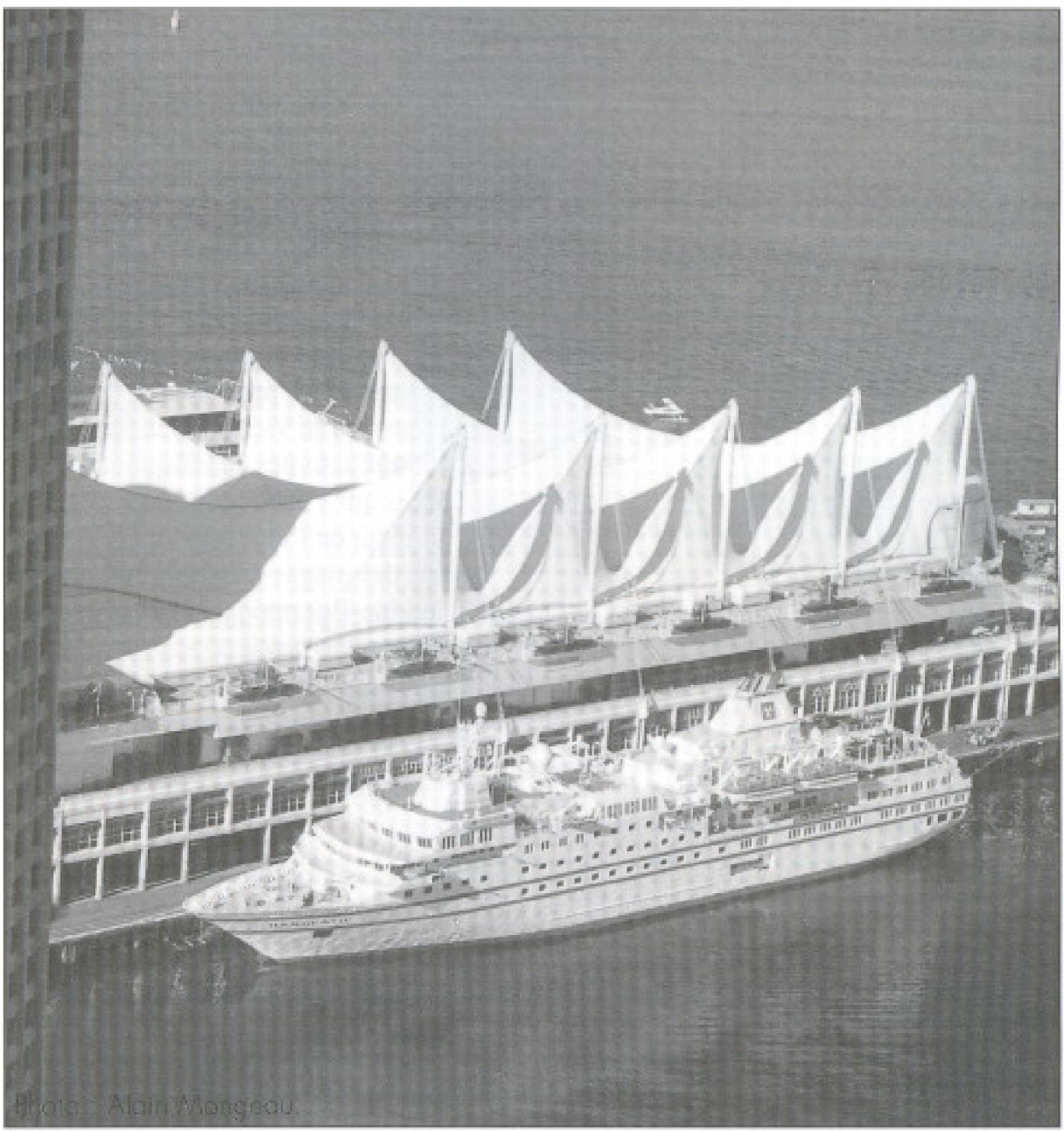


élevée de 2000 à 2005 . Il s'agit d'une tendance exponentielle dont les performances ont été plus faibles que prévues dans les deux dernières années (voir le graphique 3 ).

Depuis 1988, les touristes d'Asie sont la deuxième clientèle en importance pour la Colombie-Britannique. Compte tenu du passé, il est très probable que la tendance sera exponentielle (voir le graphique 4), On prévoit donc un taux de croissance (taux d'accroissement annuel moyen) très elevé pour les prochaines annes : $11,8 \%$ par année de 2000 à 2005.

A ce stade, il faut se demander si les taux de croissance prévus sont bien réalistes, compte tenu de fluctuations négatives possibles : la surchauffe économique qui dure depuis deux ou trois années ne sera pas éternelle, les effets de la crise financière asiatique se faisant encore sentir, particulièrement au Japon. La situation du tourisme, à moyen terme et à court terme, pourra nous éclairer sur les possibilités futures.

\section{L'évolution du tourisme à moyen terme (1990-1999)}

Le graphique 5 montre l'évolution indicielle des principales clientèles touristiques de la Colombie-Britannique de 1990 à 1999.

Nous voyons la forte progression des clientèles européennes et asiatiques de 1994 à 1997. Par contre, en 1998 et en 1999 , les différences dans l'évolution indicielle de ces trois principales clientèles sont beaucoup moins tranchées.

\section{Les mouvements saisonniers}

Malgré son climat très clément et sa position géographique, les arrivées de touristes en Colombie-Britannique sont fortement influencées par la saisonnalité, phénomène incontournable dans le domaine du tourisme (Stafford et Sarrasin, 1999). Le tableau 2 indique les différents coefficients saisonniers par mois, pour la période 1995-1999, pour les principales clientèles.

Nous pouvons constater que les coefficients des clientèles touristiques des ÉtatsUnis sont assez semblables à ceux des

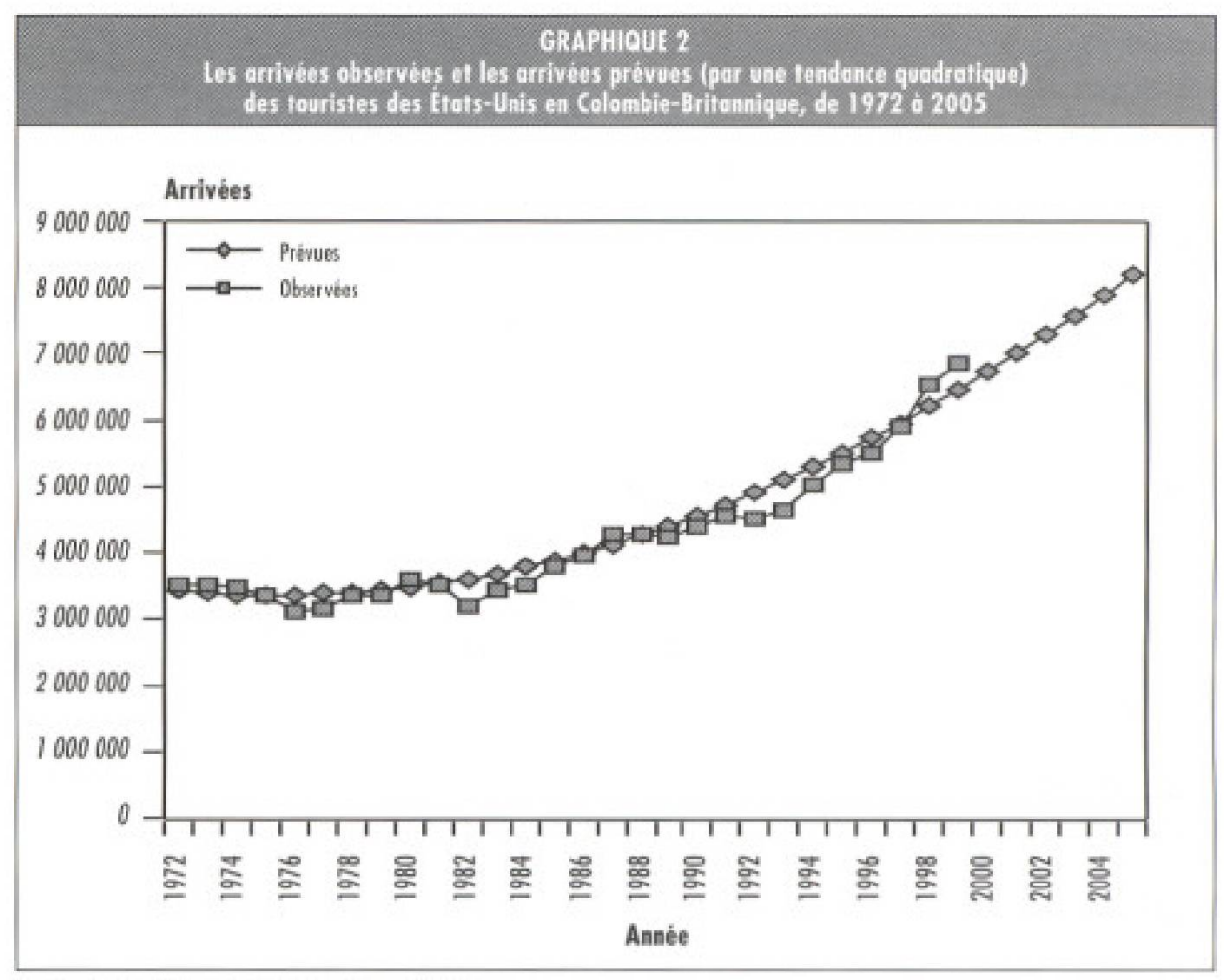

Sowce: Governement de la Colombie Btitannique:

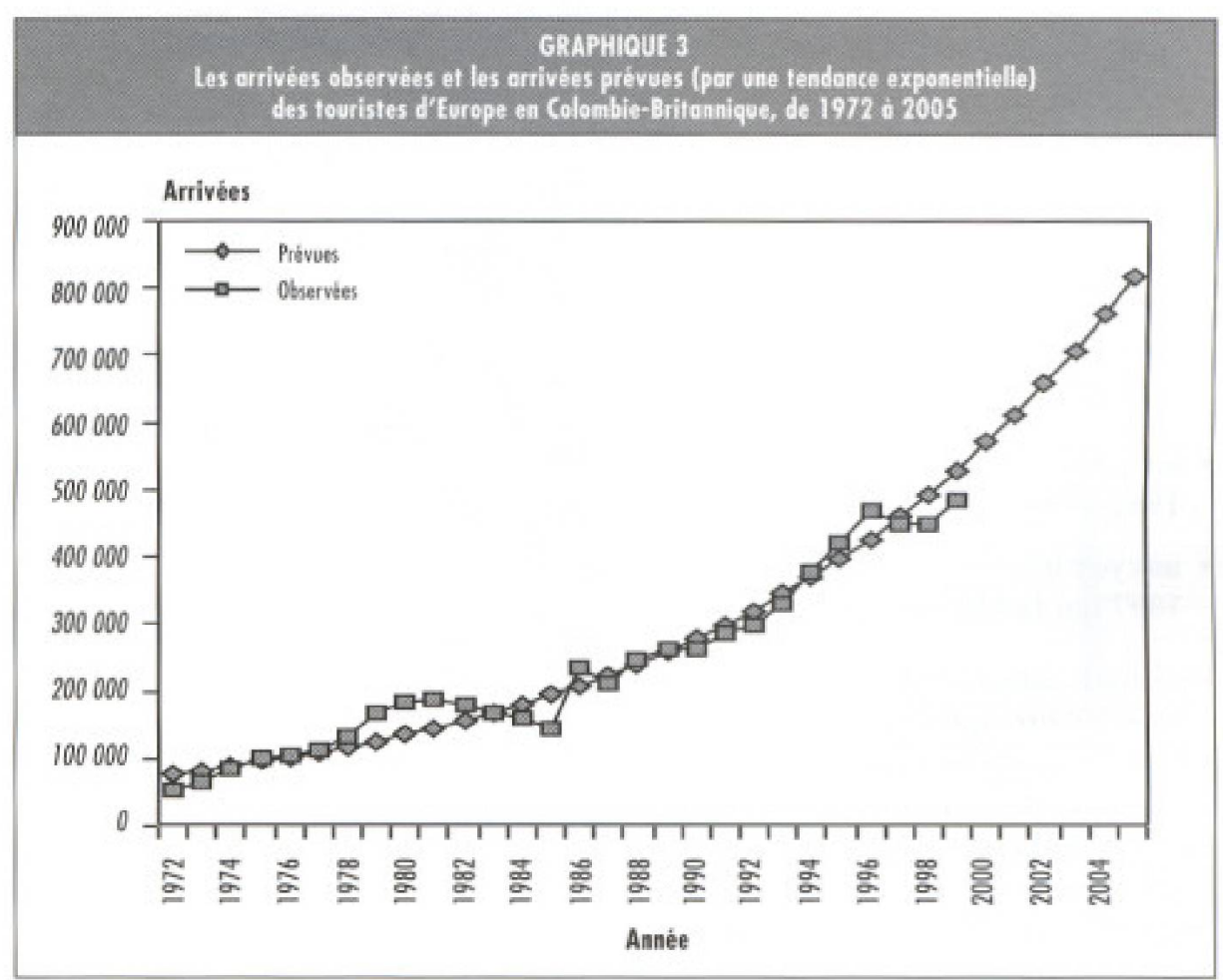

Scutce : Governement de $\mathrm{k}$ Colambie Britamique.

clientèles en provenance d'Asie. Les coefficients saisonniers des clientèles européennes se démarquent fortement des deux premières ; en effet, ils sont plus faibles de janvier à mai et d'octobre à décembre. Des mois de juin à septembre, les coefficients saisonniers des visiteurs européens sont très supérieurs à ceux des visiteurs des 
États-Unis ou de l'Asie. Le comportement saisonnier des clientèles européennes est assez traditionnel : une forte saisonnalite durant la période estivale (fin du printemps, été et début de l'automne) correspondant généralement aux congés scolaires.

\section{Les mouvements cycliques}

Pour les États-Unis au cours de la période 1977-1999, les fluctuations cycliques sont rélativement réduites avec des indices qui oscillent de 0,90 à 1,14 . Nous pouvons observer trois phases d'evolution :

- un cycle positif de cinq années : 1977 1981 ;

- un cycle négatif de quinze années, mais très faible, où la plupart des indices gravitent autour de 1 : 1982-1996:

- le début d'un cycle positif : 1997-1999.

En ce qui concerne la clientèle européenne, nous décelons:

- un cycle positif de six années: 1977 1982 :

- un cycle négatif de onze années : 1983 1993 ;

- un nouveau cycle positif qui débute en 1994.

Enfin, en ce qui a trait à la clientèle asiatique, nous constatons:

- un cycle positif de cinq années : 19771982

- un long cycle négatif de treize années: 1982-1994:

- un cycle très fort durant la période 19951997 qui faiblit par la suite.

Une étude attentive des fluctuations cycliques montre que la croissance touristique est toujours très fragile et étroitement dépendante des mouvements de l'cconomie globale. Les périodes de cycles positifs sont beaucoup moins nombreuses que celles de cycles négatifs et ces mouvements donnent l'impression que le tourisme evvolue sous la pression de a poussées $s$ qui résul tent, en grande partie, de la situation économique régionale ou mondiale, mais qui sont aussi causées par des phénomènes de mode et de saturation brusque de certains marchés.

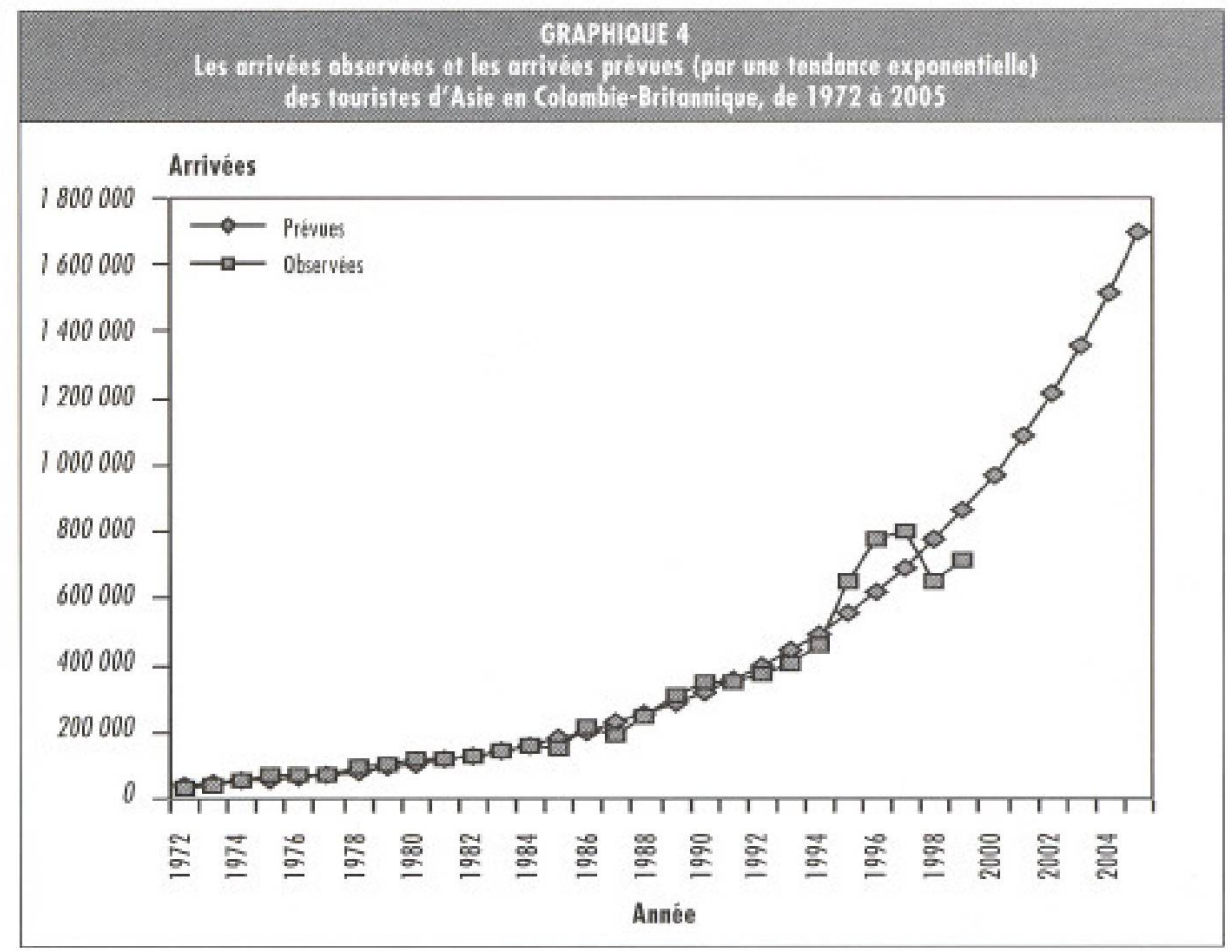

Source Gowernement de la Colombie-Britannique.

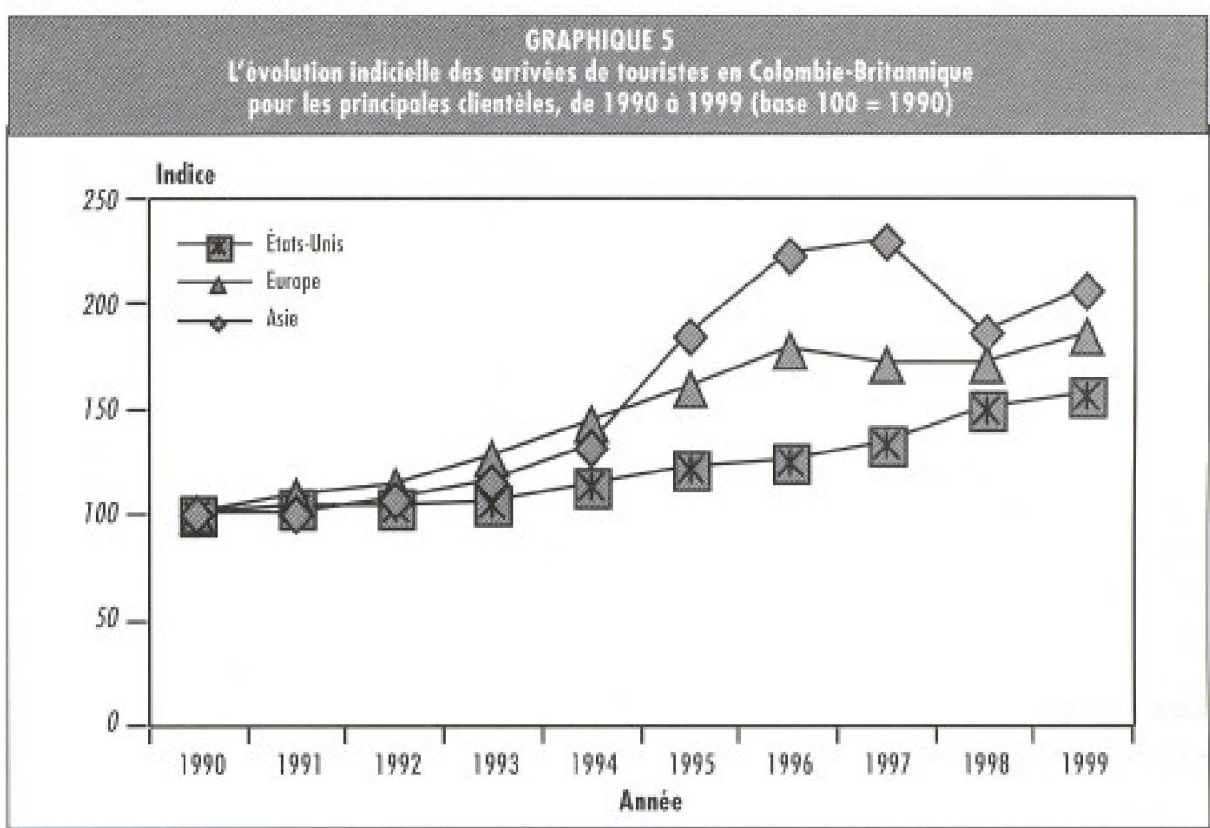

Shurce : Gouvernement de lo Colombie-Britannique.

\section{Les emplois dans le secteur du tourisme}

La croissance des emplois en tourisme a été très soutenue pendant la période 1985-1989 avec un taux d'accroissement annuel moyen de $3,5 \%$; cette croissance a été plus faible pour la période 1990-1994 (avec 1,1\%) et très faible au cours des dernieres années observes (1995-1999), avec 0,6\%.
Au cours des dix dernières années, l'augmentation du total des emplois en Colombie-Britannique aété plus rapide que l'augmentation des emplois du secteur touristique. Le tableau 3 démontre l'évolution indicielle des emplois dans $1^{*}$ ensemble des secteurs et pour le domaine du tourisme. On note que les indices sont plus élevés en tourisme seulement pour les années 1993 et 1994. Le ratio des emplois touris- 
tiques sur l'ensemble des emplois est, en moyenne, de $7,4 \%$ pendant la dernière décennie.

\section{L'importance de Vancouver}

La ville de Vancouver joue un rôle très important dans le développement du tourisme en Colombie-Britannique. D'après nos évaluations, selon les années, la part de Vancouver dans l'ensemble du tourisme en Colombie-Britannique oscille de $40 \%$ à $50 \%$ (d'après le nombre de visiteurs et les dépenses touristiques).

L'impact économique du tourisme dans la ville de Vancouver se chiffre ainsi ${ }^{2}$ en 1999 :

- effet global sur l'ensemble des industries: $6233100023 \$$;

- salaires : $2162621966 \$$;

- taxes diverses: 1428673213 \$;

- emplois : 103867 \$.

La position géographique de Vancouver - son ouverture sur le Pacifique et la proximité des États-Unis - lui confere des avantages comparatifs très importants. Cette position joue sûrement un grand rôle dans le fait que la Colombie-Britannique occupe la deuxième place dans les parts

\begin{tabular}{|c|c|c|c|}
\hline $\begin{array}{l}\text { Les coelf } \\
\text { tomistig }\end{array}$ & 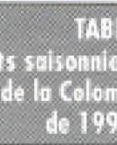 & $\begin{array}{l}12 \\
\text { les pring } \\
\text { intlininit } \\
1999\end{array}$ & $\begin{array}{l}\text { dienteles } \\
\text { nit inots }\end{array}$ \\
\hline AlMEEE & ĖATS-UNIS & EUROPE & ASIE \\
\hline Janvier & 60 & 35 & 52 \\
\hline Fèvrier & 67 & 42 & 65 \\
\hline Mors & 75 & 63 & 75 \\
\hline Avril & 78 & 58 & 83 \\
\hline Mai & 108 & 114 & 112 \\
\hline Juin & 131 & 158 & 131 \\
\hline Juillet & 170 & 208 & 175 \\
\hline Anoüt & 178 & 212 & 170 \\
\hline Septembre & 119 & 163 & 124 \\
\hline Octobre & 75 & 63 & 86 \\
\hline Novenbre & 65 & 33 & 55 \\
\hline Décembre & 71 & 50 & 71 \\
\hline
\end{tabular}

Source Gouvernemeant de lo Colombie-Britannique

\begin{tabular}{|c|c|c|c|c|c|}
\hline \multicolumn{6}{|c|}{ 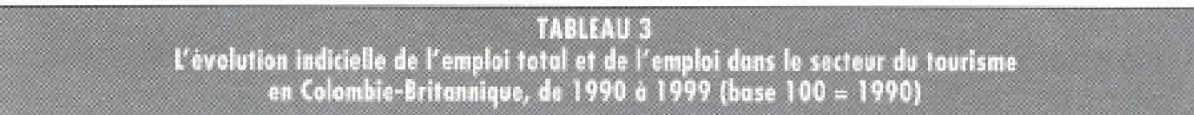 } \\
\hline ANMEE & EMPLOI TOTAL & EMPLOI TOURISME & INDICE EMPLOI TOTAL & IKDICE EMPLOI TOURISME & RATIO TOURISME - (\$) \\
\hline 1990 & 1285620 & 98840 & 100 & 100 & 7,69 \\
\hline 1991 & 1259740 & 91010 & 98 & 92 & 7,22 \\
\hline 1992 & 1259170 & 92360 & 98 & 93 & 7,33 \\
\hline 1993 & 1313100 & 103320 & 102 & 105 & 7,87 \\
\hline 1994 & 1342940 & 103440 & 104 & 105 & 7,7 \\
\hline 1995 & 1393190 & 104320 & 108 & 106 & 7,49 \\
\hline 1996 & 1408520 & 107660 & 110 & 109 & 7,64 \\
\hline 1997 & 1441670 & 110850 & 112 & 112 & 7,69 \\
\hline 1998 & 1454560 & 109490 & 113 & 111 & 7,53 \\
\hline 1999 & 1456200 & 106830 & 113 & 108 & 7,34 \\
\hline
\end{tabular}

Souce: Gouvernement de la Colombio-Britamique

de marché du tourisme au Canada (pour le nombre de touristes, les nuitées et les dépenses touristiques), après l'Ontario.

\section{Pour la suite des choses}

Depuis 1972, la Colombie-Britannique a connu un fort développement de son industrie touristique. Tranquillement cette province s'est taillé une part enviable du marché touristique canadien. $\AA$ court terme, il faut s'attendre à une certaine stagnation de la croissance des diverses clientèles, étant donné le ralentissement prévu de l'économie américaine à partir de 2001 ; par le passé, 1 industrie a vécu des baisses sensibles du nombre de visiteurs, notamment en 1976 et en 1982 et une baisse de moindre importance en 1992.

En matière touristique, la Colombie-Britannique, comme les autres provinces du Canada d'ailleurs, dépend d'un très petit nombre de pays : en premier lieu les ÉtatsUnis, puis deux ou trois pays d'Europe et trois ou quatre pays d'Asie. Un ralentissement est à craindre aux États-Unis dans les trois prochaines années (après plusieurs années de prospérité) ; l'industrie canadienne du tourisme sera donc sûrement touchée par cette conjoncture.

A moyen terme et à long terme, la situation du tourisme en Colombie-Britanni- que deviendra de plus en plus intéressante: il faut s'attendre à une croissance continue des visiteurs. L' attrait universel de la mer allié au a confort climatique " (Besancenot, 1990) demeureront des atouts irremplaçables.

Jean Stafford est professeur au Département d'études urbaines et touristiques de IUUQAM.

Bruno Sarrasin est chargé de cours au Département d'études urbaines et touristiques de l'UOAM.

\section{Notes}

1 Les données utilisées sont tirées du site Internet : www.gov,bc.ca

2 Vancouver's Tourism Economic Assessment Model : www.tourism-vancouver,org

\section{Bibliographie}

Besancenot, Jean-Pierre (1990), Clinat et tourisme, Paris, Masson.

Stafford, Jean, et Sarrasin, Bruno (1999), « La saisonnalité dans les hôtels du Québec : problèmes ou opportunités $\%$, Téoros, 18 (1).

www.tourism-vancouver.org www.bestats.gov.bc.ca 